\title{
Existence results for a class of nonlocal problems involving p-Laplacian
}

Yang Yang ${ }^{1 *}$ and Jihui Zhang ${ }^{2}$

* Correspondence: yynjnu@126. com

${ }^{1}$ School of Science, Jiangnan University, Wuxi, 214122, People's Republic of China

Full list of author information is available at the end of the article

\section{Abstract}

This paper is concerned with the existence of solutions to a class of p-Kirchhoff type equations with Neumann boundary data as follows:

$$
\begin{cases}-\left[M\left(\int_{\Omega}|\nabla u|^{p} \mathrm{~d} x\right)\right]^{p-1} \Delta_{p} u=f(x, u) & \text { in } \Omega ; \\ \frac{\partial u}{\partial v}=0, & \text { on } \partial \Omega .\end{cases}
$$

By means of a direct variational approach, we establish conditions ensuring the existence and multiplicity of solutions for the problem.

Keywords: Nonlocal problems, Neumann problem, p-Kirchhoff's equation

\section{Introduction}

In this paper, we deal with the nonlocal p-Kirchhoff type of problem given by:

$$
\begin{cases}-\left[M\left(\int_{\Omega}|\nabla u|^{p} \mathrm{~d} x\right)\right]^{p-1} \Delta_{p} u=f(x, u) & \text { in } \Omega ; \\ \frac{\partial u}{\partial v}=0, & \text { on } \partial \Omega\end{cases}
$$

where $\Omega$ is a smooth bounded domain in $\mathbf{R}^{\mathbf{N}}, 1<p<N, v$ is the unit exterior vector on $\partial \Omega, \Delta_{p}$ is the $p$-Laplacian operator, that is, $\Delta_{p} u=\operatorname{div}\left(|\nabla u|^{p-2} \nabla u\right)$, the function $M$ : $\mathbf{R}^{+} \rightarrow \mathbf{R}^{+}$is a continuous function and there is a constant $m_{0}>0$, such that

$$
\left(M_{0}\right) \quad M(t) \geq m_{0} \text { for all } t \geq 0 .
$$

$f(x, t): \bar{\Omega} \times \mathbf{R} \rightarrow \mathbf{R}$ is a continuous function and satisfies the subcritical condition:

$$
|f(x, t)| \leq C\left(|t|^{q-1}+1\right), \quad \text { for some } \quad p<q<p^{*}=\left\{\begin{array}{l}
\frac{N p}{N-p}, N \geq 3 \\
+\infty, N=1,2
\end{array}\right.
$$

where $C$ denotes a generic positive constant.

Problem (1.1) is called nonlocal because of the presence of the term $M$, which implies that the equation is no longer a pointwise identity. This provokes some mathematical difficulties which makes the study of such a problem particulary interesting. This problem has a physical motivation when $p=2$. In this case, the operator $M$ $\left(\int_{\Omega}|\nabla u|^{2} \mathrm{~d} x\right) \Delta u$ appears in the Kirchhoff equation which arises in nonlinear vibrations, namely 


$$
\begin{cases}u_{t t}-M\left(\int_{\Omega}|\nabla u|^{2} \mathrm{~d} x\right) \Delta u=f(x, u), & \text { in } \Omega \times(0, T) \\ u=0, & \text { on } \partial \Omega \times(0, T) ; \\ u(x, 0)=u_{0}(x), & u_{t}(x, 0)=u_{1}(x) .\end{cases}
$$

P-Kirchhoff problem began to attract the attention of several researchers mainly after the work of Lions [1], where a functional analysis approach was proposed to attack it. The reader may consult [2-8] and the references therein for similar problem in several cases.

This work is organized as follows, in Section 2, we present some preliminary results and in Section 3 we prove the main results.

\section{Preliminaries}

By a weak solution of (1.1), then we say that a function $u \varepsilon W^{1, p}(\Omega)$ such that

$$
\left[M\left(\int_{\Omega}|\nabla u|^{p} \mathrm{~d} x\right)\right]^{p-1} \int_{\Omega}|\nabla u|^{p-2} \nabla u \nabla \varphi \mathrm{d} x=\int_{\Omega} f(x, u) \varphi \mathrm{d} x, \quad \text { for all } \quad \varphi \in W^{1, p}(\Omega)
$$

So we work essentially in the space $W^{1, p}(\Omega)$ endowed with the norm

$$
\|u\|=\left(\int_{\Omega}\left(|\nabla u|^{p}+|u|^{p}\right) \mathrm{d} x\right)^{\frac{1}{p}},
$$

and the space $W^{1, p}(\Omega)$ may be split in the following way. Let $W_{c}=\langle 1\rangle$, that is, the subspace of $W^{1, p}(\Omega)$ spanned by the constant function 1 , and $W_{0}=\left\{z \in W^{1, p}(\Omega), \int_{\Omega} z=0\right\}$, which is called the space of functions of $W^{1, p}(\Omega)$ with null mean in $\Omega$. Thus

$$
W^{1, p}(\Omega)=W_{0} \oplus W_{c} .
$$

As it is well known the Poincarés inequality does not hold in the space $W^{1, p}(\Omega)$. However, it is true in $W_{0}$.

Lemma 2.1 [8] (Poincaré-Wirtinger's inequality) There exists a constant $\eta>0$ such that $\int_{\Omega}|z|^{p} \mathrm{~d} x \leq \eta \int_{\Omega}|\nabla z|^{p} \mathrm{~d} x$ for all $z \in W_{0}$.

Let us also recall the following useful notion from nonlinear operator theory. If $X$ is a Banach space and $A: X \rightarrow X^{*}$ is an operator, we say that $A$ is of type $\left(S_{+}\right)$, if for every sequence $\left\{x_{n}\right\}_{n \geq 1} \subseteq X$ such that $x_{n}-x$ weakly in $X$, and $\limsup _{n \rightarrow \infty}\left\langle A\left(x_{n}\right), x_{n}-x\right\rangle \leq 0$. we have that $x_{n} \rightarrow x$ in $X$.

Let us consider the map $A: W^{1, p}(\Omega) \rightarrow W^{1, p}(\Omega)^{*}$ corresponding to $-\Delta_{p}$ with Neumann boundary data, defined by

$$
\langle A(u), v\rangle=\int_{\Omega}|\nabla u|^{p-2} \nabla u \nabla v \mathrm{~d} x, \quad \forall u, v \in W^{1, p}(\Omega) .
$$

We have the following result:

Lemma $2.2[9,10]$ The map $A: W^{1, p}(\Omega) \rightarrow W^{1, p}(\Omega) *$ defined by $(2.1)$ is continuous and of type $\left(S_{+}\right)$.

In the next section, we need the following definition and the lemmas.

Definition 2.1. Let $E$ be a real Banach space, and $D$ an open subset of E. Suppose that a functional $J: D \rightarrow R$ is Fréchet differentiable on $D$. If $x_{0} \in D$ and the Fréchet derivative $J^{\prime}\left(x_{0}\right)=0$, then we call that $x_{0}$ is a critical point of the functional $J$ and $c=$ $J\left(x_{0}\right)$ is a critical value of $J$. 
Definition 2.2. For $J \in C^{1}(E, \mathbf{R})$, we say $J$ satisfies the Palais-Smale condition (denoted by (PS)) if any sequence $\left\{u_{n}\right\} \subset E$ for which $J\left(u_{n}\right)$ is bounded and $J^{\prime}\left(u_{n}\right) \rightarrow 0$ as $n \rightarrow \infty$ possesses a convergent subsequence.

Lemma 2.3 [11] Let $X$ be a Banach space with a direct sum decomposition $X=X_{1} \oplus$ $X_{2}$, with $k=\operatorname{dim} X_{2}<\infty$, let $J$ be a $C^{1}$ function on $X$, satisfying (PS) condition. Assume that, for some $r>0$,

$$
\begin{array}{ll}
J(u) \leq 0 \text { for } u \in X_{1}, & \|u\| \leq r ; \\
J(u) \geq 0 \text { for } u \in X_{2}, & \|u\| \leq r .
\end{array}
$$

Assume also that $J$ is bounded below and $\inf _{X} J<0$. Then $J$ has at least two nonzero critical points.

Lemma 2.4 [12]Let $X=X_{1} \oplus X_{2}$, where $X$ is a real Banach space and $X_{2} \neq\{0\}$, and is finite dimensional. Suppose $J \in C^{1}(X, R)$ satisfies (PS) and

(i) there is a constant $\alpha$ and a bounded neighborhood $D$ of 0 in $X_{2}$ such that $\left.J\right|_{\partial D} \leq \alpha$ and,

(ii) there is a constant $\beta>\alpha$ such that $\left.J\right|_{X_{1}} \geq \beta$,

then J possesses a critical value $c \geq \beta$, moreover, $c$ can be characterized as

$$
c=\inf _{h \in \Gamma} \max _{u \in \bar{D}} J(h(u)) .
$$

where $\Gamma=\{h \in C(\bar{D}, X) \mid h=i d$ on $\partial D\}$.

Definition 2.3. For $J \in C^{1}(E, \mathbf{R})$, we say J satisfies the Cerami condition (denoted by $(C))$ if any sequence $\left\{u_{n}\right\} \subset E$ for which $J\left(u_{n}\right)$ is bounded and $\left(1\left\|u_{n}\right\|\right) J\left(u_{n}\right) \| \rightarrow 0$ as $n \rightarrow \infty$ possesses a convergent subsequence.

Remark 2.1 If $J$ satisfies the (C) condition, Lemma 2.4 still holds.

In the present paper, we give an existence theorem and a multiplicity theorem for problem (1.1). Our main results are the following two theorems.

Theorem 2.1 If following hold:

$\left(F_{0}\right) \quad 0 \leq \lim _{|u| \rightarrow 0} \frac{p F(x, u)}{|u|^{p}}<\frac{m_{0}^{p-1}}{\eta}$ a.e. $x \in \Omega$, where $F(x, u)=\int_{0}^{u} f(x, s) d s$, $\eta$ appears in

Lemma 2.1;

$\left(F_{1}\right) \lim _{|u| \rightarrow \infty} \frac{p F(x, u)}{|u|^{p}} \leq 0$ a.e. $x \in \Omega$;

$\left(F_{2}\right) \lim _{|u| \rightarrow \infty} \int_{\Omega} F(x, u) \mathrm{d} x=-\infty$.

Then the problem (1.1) has least three distinct weak solutions in $W^{1, p}(\Omega)$.

Theorem 2.2 If the following hold:

$\left(M_{1}\right)$ The function $M$ that appears in the classical Kirchhoff equation satisfies $\widehat{M}(t) \leq(M(t))^{p-1}$ ffor all $t \geq 0$, where $\widehat{M}(t)=\int_{0}^{t}[M(s)]^{p-1} d s ;$

$\left(F_{3}\right) f(x, u) u>0$ for all $u \neq 0$;

$\left(F_{4}\right) \lim _{|u| \rightarrow \infty} \frac{p F(x, u)}{|u|^{\mid}}=0$ a.e. $x \in \Omega$;

$\left(F_{5}\right) \lim _{|u| \rightarrow \infty}(f(x, u) u-p F(x, u))=-\infty$.

Then the problem (1.1) has at least one weak solution in $W^{1, p}(\Omega)$.

Remark 2.2 We exhibit now two examples of nonlinearities that fulfill all of our hypotheses

$$
f(x, u)=\frac{m_{0}^{p-1}}{2 \eta}|u|^{p-2} u-|u|^{q-2} u,
$$


hypotheses $\left(F_{0}\right),\left(F_{1}\right),\left(F_{2}\right)$ and $(1.2)$ are clearly satisfied.

$$
f(x, u)=\arctan u+\frac{u}{1+u^{2}},
$$

hypotheses $\left(F_{3}\right),\left(F_{4}\right)$ and $\left(F_{5}\right)$ and (1.2) are clearly satisfied.

\section{Proofs of the theorems}

Let us start by considering the functional $J: W^{1, p}(\Omega) \rightarrow \mathbf{R}$ given by

$$
J(u)=\frac{1}{p} \widehat{M}\left(\int_{\Omega}|\nabla u|^{p} \mathrm{~d} x\right)-\int_{\Omega} F(x, u) \mathrm{d} x .
$$

Proof of Theorem 2.1 By $\left(F_{0}\right)$, we know that $f(x, 0)=0$, and hence $u(x)=0$ is a solution of (1.1).

To complete the proof we prove the following lemmas.

Lemma 3.1 Any bounded (PS) sequence of J has a strongly convergent subsequence.

Proof: Let $\left\{u_{n}\right\}$ be a bounded (PS) sequence of $J$. Passing to a subsequence if necessary, there exists $u \in W^{1, p}(\Omega)$ such that $u_{n} \rightarrow u$. From the subcritical growth of $f$ and the Sobolev embedding, we see that

$$
\int_{\Omega} f\left(x, u_{n}\right)\left(u_{n}-u\right) \mathrm{d} x \rightarrow 0 .
$$

and since $J^{\prime}\left(u_{n}\right)\left(u_{n}-u\right) \rightarrow 0$, we conclude that

$$
\left[M\left(\int_{\Omega}\left|\nabla u_{n}\right|^{p} \mathrm{~d} x\right)\right]^{p-1} \int_{\Omega}\left|\nabla u_{n}\right|^{p-2} \nabla u_{n} \nabla\left(u_{n}-u\right) \mathrm{d} x \rightarrow 0 .
$$

In view of condition $\left(M_{0}\right)$, we have

$$
\int_{\Omega}\left|\nabla u_{n}\right|^{p-2} \nabla u_{n} \nabla\left(u_{n}-u\right) \mathrm{d} x \rightarrow 0 .
$$

Using Lemma 2.2, we have $u_{n} \rightarrow u$ as $n \rightarrow \infty$. ㅁ

Lemma 3.2 If condition $\left(M_{0}\right),\left(F_{1}\right)$ and $\left(F_{2}\right)$ hold, then $\lim _{\|u\| \rightarrow \infty} J(u)=+\infty$.

Proof: If there are a sequence $\left\{u_{n}\right\}$ and a constant $C$ such that $\left\|u_{n}\right\| \rightarrow \infty$ as $n \rightarrow \infty$, and $J\left(u_{n}\right) \leq C(n=1,2 \cdots)$, let $v_{n}=\frac{u_{n}}{\left\|u_{n}\right\|}$, then there exist $v_{0} \in W^{1, p}(\Omega)$ and a subsequence of $\left\{v_{n}\right\}$, we still note by $\left\{v_{n}\right\}$, such that $v_{n} \rightarrow v_{0}$ in $W^{1, p}(\Omega)$ and $v_{n} \rightarrow v_{0}$ in $L^{p}(\Omega)$.

For any $\varepsilon>0$, by $\left(F_{1}\right)$, there is a $H>0$ such that $F(x, u) \leq \frac{\varepsilon}{p}|u|^{p}$ for all $|u| \geq H$ and a. e. $x \in \Omega$, then there exists a constant $C>0$ such that $F(x, u) \leq \frac{\varepsilon}{p}|u|^{p}+C$ for all $u \in R$, and a.e. $x \in \Omega$, Consequently

$$
\begin{aligned}
\frac{C}{\left\|u_{n}\right\|^{p}} & \geq \frac{J\left(u_{n}\right)}{\left\|u_{n}\right\|^{p}}=\frac{1}{\left\|u_{n}\right\|^{p}}\left(\frac{1}{p} \widehat{M}\left(\int_{\Omega}\left|\nabla u_{n}\right|^{p} \mathrm{~d} x\right)-\int_{\Omega} F\left(x, u_{n}\right) \mathrm{d} x\right) \\
& \geq \frac{1}{p} m_{0}^{p-1} \int_{\Omega}\left|\nabla v_{n}\right|^{p} \mathrm{~d} x-\frac{\varepsilon}{p} \int_{\Omega}\left|v_{n}\right|^{p} \mathrm{~d} x-\frac{C|\Omega|}{\left\|u_{n}\right\|^{p}} \\
& =\frac{1}{p} m_{0}^{p-1}-\left(\frac{1}{p} m_{0}^{p-1}+\frac{\varepsilon}{p}\right) \int_{\Omega}\left|v_{n}\right|^{p} \mathrm{~d} x-\frac{C|\Omega|}{\left\|u_{n}\right\|^{p}} .
\end{aligned}
$$

It implies $\int_{\Omega}\left|v_{0}\right|^{p} \mathrm{~d} x \geq 1$. On the other hand, by the weak lower semi-continuity of the norm, one has 


$$
\begin{aligned}
& \| v_{0}|| \leq \liminf _{n \rightarrow \infty}|| v_{n}||=1 . \\
& \text { Hence } \int_{\Omega}\left|\nabla v_{0}\right|^{p} \mathrm{~d} x=0 \text {, so }\left|v_{0}(x)\right|=\text { constant } \neq 0 \text { a.e. } x \in \Omega \text {. By }\left(F_{2}\right), \\
& \lim _{\left|u_{n}\right| \rightarrow \infty} \int_{\Omega} F\left(x, u_{n}\right) \mathrm{d} x \rightarrow-\infty \text {. Hence } \\
& C \geq J\left(u_{n}\right)=\frac{1}{p} \widehat{M}\left(\int_{\Omega}\left|\nabla u_{n}\right|^{p} \mathrm{~d} x\right)-\int_{\Omega} F\left(x, u_{n}\right) \mathrm{d} x \\
& \geq-\int_{\Omega} F\left(x, u_{n}\right) \mathrm{d} x \rightarrow+\infty \text { as } n \rightarrow \infty .
\end{aligned}
$$

This is a contradiction. Hence $J$ is coercive on $W^{1, p}(\Omega)$, bounded from below, and satisfies the (PS) condition. $\square$

By Lemma 3.1 and 3.2, we know that $J$ is coercive on $W^{1, p}(\Omega)$, bounded from below, and satisfies the (PS) condition. From condition $\left(F_{0}\right)$, we know, there exist $r>0, \varepsilon>0$ such that

$$
0 \leq F(x, u) \leq\left(\frac{m_{0}^{p-1}}{p \eta}-\varepsilon\right)|u|^{p}, \quad \text { for }|u| \leq r .
$$

If $u \in W_{c}$, for $\|u\| \leq \rho_{1}$, then $|u| \leq r$, we have

$$
\begin{aligned}
J(u) & =\frac{1}{p} \widehat{M}\left(\int_{\Omega}|\nabla u|^{p} \mathrm{~d} x\right)-\int_{\Omega} F(x, u) \mathrm{d} x \\
& =-\int_{\Omega} F(x, u) \mathrm{d} x \leq 0 .
\end{aligned}
$$

If $u \in W_{0}$, then from condition $\left(F_{0}\right)$ and (1.2), we have

$$
F(x, u) \leq\left(\frac{m_{0}^{p-1}}{p \eta}-\varepsilon\right)|u|^{p}+C|u|^{q}, \quad \text { for } \quad u \in R, \quad q \in\left(p, p^{*}\right) .
$$

Noting that

$$
\int_{\Omega}|u|^{p} \mathrm{~d} x \leq \eta \int_{\Omega}|\nabla u|^{p} \mathrm{~d} x, \quad u \in W_{0}
$$

we can obtain

$$
\begin{aligned}
J(u) & =\frac{1}{p} \widehat{M}\left(\int_{\Omega}|\nabla u|^{p} \mathrm{~d} x\right)-\int_{\Omega} F(x, u) \mathrm{d} x \\
& \geq \frac{1}{p} m_{0}^{p-1} \int_{\Omega}|\nabla u|^{p} \mathrm{~d} x-\frac{m_{0}^{p-1}}{p \eta} \int_{\Omega}|u|^{p} \mathrm{~d} x+\varepsilon \int_{\Omega}|u|^{p} \mathrm{~d} x-C \int_{\Omega}|u|^{q} \mathrm{~d} x \\
& \geq C \varepsilon\left\|\left.u\right|^{p}-C C_{1}|| u\right\|^{q} .
\end{aligned}
$$

Choose $\|u\|=\rho_{2}$ small enough, such that $J(u) \geq 0$ for $\|u\| \leq \rho_{2}$ and $u \in W_{0}$. Now choose $\rho=\min \left\{\rho_{1}, \rho_{2}\right\}$, then, we have

$$
\begin{aligned}
& J(u) \leq 0 \text { for } u \in W_{c}, \quad\|u\| \leq \rho ; \\
& J(u) \leq 0 \text { for } u \in W_{0}, \quad\|u\| \leq \rho .
\end{aligned}
$$


If $\inf \left\{(u), u \in W^{1, p}(\Omega)\right\}=0$, then all $u \in W_{c}$ with $\|u\| \leq \rho$ are minimum of $J$, which implies that $J$ has infinite critical points. If $\inf \left\{J(u), u \in W^{1, p}(\Omega)\right\}<0$ then by Lemma $2.3, J$ has at least two nontrivial critical points. Hence problem (1.1) has at least two nontrivial solutions in $W^{1, p}(\Omega)$, Therefore, problem (1.1) has at least three distinct solutions in $W^{1, p}(\Omega)$. $\square$

Proof of Theorem 2.2. We divide the proof into several lemmas.

Lemma 3.3 If condition $\left(F_{3}\right)$ and $\left(F_{5}\right)$ hold, then $\left.J\right|_{W_{c}}$ is anticoercive. (i.e. we have that $J(u) \rightarrow-\infty$, as $|u| \rightarrow \infty, u \in R$.)

Proof: By virtue of hypothesis $\left(F_{5}\right)$, for any given $L>0$, we can find $R_{1}=R_{1}(L)>0$ such that

$$
F(x, u) \geq \frac{1}{p} L+\frac{1}{p} f(x, u) u, \quad \text { for a.e. } x \in \Omega, \quad|u|>R_{1} .
$$

Thus, using hypothesis $\left(F_{3}\right)$, we have

$$
F(x, u) \geq \frac{1}{p} L-C, \text { for a.e. } x \in \Omega u \in \mathbf{R}
$$

So

$$
\int_{\Omega} F(x, u) \mathrm{d} x \geq \frac{1}{p} L|\Omega|-C|\Omega| .
$$

Since $L>0$ is arbitrary, it follows that

$$
\int_{\Omega} F(x, u) \mathrm{d} x \rightarrow \infty, \quad \text { as } \quad|u| \rightarrow \infty,
$$

and so

$$
\left.J(u)\right|_{W_{C}}=-\int_{\Omega} F(x, u) \mathrm{d} x \rightarrow-\infty, \quad \text { as } \quad|u| \rightarrow \infty .
$$

This proves that $\left.J\right|_{W_{c}}$ is anticoercive.

Lemma 3.4 If hypothesis $\left(F_{4}\right)$ holds, then $\left.J\right|_{W_{0}} \geq-\infty$.

Proof: For a given $0<\varepsilon<m_{0}^{p-1}$, we can find $C_{\varepsilon}>0$ such that $F(x, u) \leq \frac{\varepsilon}{p \eta}|u|^{p}+C_{\varepsilon}$ for a.e. $x \in \Omega$ all $u \in \mathbf{R}$. Then

$$
\begin{aligned}
\left.J(u)\right|_{u \in W_{0}} & =\frac{1}{p} \widehat{M}\left(\int_{\Omega}|\nabla u|^{p} \mathrm{~d} x\right)-\int_{\Omega} F(x, u) \mathrm{d} x \\
& \geq \frac{1}{p} m_{0}^{p-1} \int_{\Omega}|\nabla u|^{p} \mathrm{~d} x-\frac{m_{0}^{p-1}}{p \eta} \int_{\Omega}|u|^{p} \mathrm{~d} x-C|\Omega| \\
& \geq-C|\Omega| .
\end{aligned}
$$

then $\left.J\right|_{W_{0}} \geq-\infty$. $\square$

Lemma 3.5 If condition $\left(F_{4}\right)\left(F_{5}\right)$ hold, then J satisfies the $(C)$ condition.

Proof: Let $\left\{u_{n}\right\}_{n \geq 1} \subseteq W^{1, p}(\Omega)$ be a sequence such that

$$
\left|J\left(u_{n}\right)\right| \leq M_{1}, \quad \forall n \geq 1 .
$$

with some $M_{1}>0$ and

$$
\left(1+\left\|u_{n}\right\|\right) J^{\prime}\left(u_{n}\right) \rightarrow 0, \quad \text { in } \quad W^{1, p}(\Omega)^{*} \quad \text { as } \quad n \rightarrow \infty .
$$


We claim that the sequence $\left\{u_{n}\right\}$ is bounded. We argue by contradiction. Suppose that $\|u\| \rightarrow+\infty$, as $n \rightarrow \infty$, we set $v_{n}=\frac{u_{n}}{\left\|u_{n}\right\|}, \forall n \geq 1$. Then $\left\|v_{n}\right\|=1$ for all $n \geq 1$ and so, passing to a subsequence if necessary, we may assume that

$$
\begin{aligned}
& v_{n} \rightarrow v \text { in } W^{1, p}(\Omega) ; \\
& v_{n} \rightarrow v \text { in } L^{p}(\Omega) .
\end{aligned}
$$

from (3.2), we have $\forall h \in W^{1, p}(\Omega)$

$$
\left.\left|\left[M\left(\int_{\Omega}\left|\nabla u_{n}\right|^{p} \mathrm{~d} x\right)\right]^{p-1} \int_{\Omega}\right| \nabla v_{n}\right|^{p-2} \nabla v_{n} \nabla h \mathrm{~d} x-\int_{\Omega} \frac{f\left(x, u_{n}\right) h}{\left\|u_{n}\right\|^{p-1}} \mathrm{~d} x \mid \leq \frac{\varepsilon_{n}}{1+\left\|u_{n}\right\|} \frac{\|h\|}{\left\|u_{n}\right\|^{p-1}}
$$

with $\varepsilon_{n} \downarrow 0$.

In (3.3), we choose $h=v_{n}-v \in W^{1, p}(\Omega)$, note that by virtue of hypothesis $\left(F_{4}\right)$, we have

$$
\frac{f\left(x, u_{n}\right)}{\left\|u_{n}\right\|^{p-1}} \rightarrow 0 \quad \text { in } \quad L^{p^{\prime}}(\Omega)
$$

where $\frac{1}{p}+\frac{1}{p^{\prime}}=1$.

So we have

$$
\left[M\left(\int_{\Omega}\left|\nabla u_{n}\right|^{p} \mathrm{~d} x\right)\right]^{p-1} \int_{\Omega}\left|\nabla v_{n}\right|^{p-2} \nabla v_{n} \nabla\left(v_{n}-v\right) \mathrm{d} x \rightarrow 0 .
$$

Since $M(t)>m_{0}$ for all $t \geq 0$, so we have

$$
\int_{\Omega}\left|\nabla v_{n}\right|^{p-2} \nabla v_{n} \nabla\left(v_{n}-v\right) \mathrm{d} x \rightarrow 0 .
$$

Hence, using the $\left(S_{+}\right)$property, we have $v_{n} \rightarrow v$ in $W^{1, p}(\Omega)$ with $\|v\|=1$, then $v \neq 0$. Now passing to the limit as $n \rightarrow \infty$ in (3.3), we obtain

$$
\int_{\Omega}|\nabla v|^{p-2} \nabla v \nabla h \mathrm{~d} x \rightarrow 0, \forall h \in W^{1, p}(\Omega),
$$

then $v=\xi \in R$. Then $\left|u_{n}(x)\right| \rightarrow+\infty$ as $n \rightarrow+\infty$. Using hypothesis $\left(F_{5}\right)$, we have $f(x$, $\left.u_{n}(x)\right) u_{n}(x)-p F\left(x, u_{n}(x)\right) \rightarrow-\infty$ for a.e $x \in \Omega$.

Hence by virtue of Fatou's Lemma, we have

$$
\int_{\Omega} f\left(x, u_{n}\right) u_{n}-p F\left(x, u_{n}\right) \mathrm{d} x \rightarrow-\infty, \quad \text { as } \quad n \rightarrow+\infty .
$$

From (3.1), we have

$$
\widehat{M}\left(\int_{\Omega}\left|\nabla u_{n}\right|^{p}\right) \mathrm{d} x-p \int_{\Omega} F\left(x, u_{n}\right) \mathrm{d} x \geq-p M_{1}, \quad \forall n \geq 1 .
$$

From (3.2), we have

$$
\left.\left|\left[M\left(\int_{\Omega}\left|\nabla u_{n}\right|^{p} \mathrm{~d} x\right)\right]^{p-1} \int_{\Omega}\right| \nabla u_{n}\right|^{p-2} \nabla u_{n} \nabla h \mathrm{~d} x-\int_{\Omega} f\left(x, u_{n}\right) h \mathrm{~d} x \mid \leq \frac{\varepsilon_{n}|| h \| \mid}{1+\left\|u_{n}\right\|} \forall h \in W^{1, p}(\Omega) .
$$


With $\varepsilon_{n} \downarrow 0$. So choosing $h=u_{n} \in W^{1, p}(\Omega)$, we obtain

$$
-\left[M\left(\int_{\Omega}\left|\nabla u_{n}\right|^{p} \mathrm{~d} x\right)\right]^{p-1} \int_{\Omega}\left|\nabla u_{n}\right|^{p} \mathrm{~d} x+\int_{\Omega} f\left(x, u_{n}\right) u_{n} \mathrm{~d} x \geq-\varepsilon_{n} .
$$

Adding (3.5) and (3.6), noting that $\widehat{M}(t) \leq(M(t))^{p-1} t$ for all $t \geq 0$, we obtain

$$
\int_{\Omega}\left(f\left(x, u_{n}\right) u_{n}-p F\left(x, u_{n}\right)\right) \mathrm{d} x \geq-M_{2}, \quad \forall n \geq 1,
$$

comparing (3.4) and (3.7), we reach a contradiction. So $\left\{u_{n}\right\}$ in bounded in $W^{1, p}(\Omega)$. Similar with the proof of Lemma 3.1, we know that $J$ satisfied the $(C)$ condition. $\square$

Sum up the above fact, from Lemma 2.4 and Remark 2.1, Theorem 2.2 follows from the Lemma 3.3 to 3.5 .

\section{Acknowledgements}

The authors would like to thank the referees for their valuable comments and suggestions.

This study was supported by NSFC (No. 10871096), the Fundamental Research Funds for the Central Universities (No. JUSRP11118)

\section{Author details}

${ }^{1}$ School of Science, Jiangnan University, Wuxi, 214122, People's Republic of China ${ }^{2}$ Institute of Mathematics, School of Mathematics Science, Nanjing Normal University, Nanjing, 210097, People's Republic of China

\section{Authors' contributions}

All authors read and approved the final manuscript.

\section{Competing interests}

The authors declare that they have no competing interests.

Received: 7 January 2011 Accepted: 11 October 2011 Published: 11 October 2011

\section{References}

1. Lions, JL: On some equations in boundary value problems of mathematical physics. In Contemporary developments in Continuum Mechanics and Partial Differential equations (Proc. Internat. Sympos., Inst. Mat., Univ. fed. Rio de Janeiro, Riio de Janeiro, 1977), North-Holland Mathematics Studies, vol. 30, pp. 284-346.North-Holland, Amsterdam (1978)

2. Alves, CO, Corrêa, FJSA, Ma, TF: Positive solutions for a quasilinear elliptic equation of Kirchhoff type. Comput Math Appl. 49(1), 85-93 (2005). doi:10.1016/j.camwa.2005.01.008

3. Ma, TF, Rivera, JEM: Positive solutions for a nonlinear elliptic transmission problem. Appl Math Lett. 16(2), 243-248 (2003). doi:10.1016/S0893-9659(03)80038-1

4. Corrêa, FJSA, Figueiredo, GM: On an elliptic equation of p-Kirchhoff type via variational methods. Bull Austral Math Soc. 74, 263-277 (2006). doi:10.1017/S000497270003570X

5. Perera, K, Zhang, ZT: Nontrivial solutions of Kirchhoff-type problems via the Yang-index. J Differ Equ. 221(1), 246-255 (2006). doi:10.1016/j.jde.2005.03.006

6. Zhang, ZT, Perera, K: Sign-changing solutions of Kirchhoff type problems via invariant sets of descent flow. J Math Anal Appl. 317(2), 456-463 (2006). doi:10.1016/j.jmaa.2005.06.102

7. Mao, AM, Zhang, ZT: Sign-changing and multiple solutions of Kirchhoff type problems without the P.S. condition. Nonlinear Anal. 70, 1275-1287 (2009). doi:10.1016/.j.na.2008.02.011

8. Corrêa, FJSA, Nascimento, RG: On a nonlocal elliptic system of p-Kirchhoff type under Neumann boundary condition. Math Comput Model. 49, 598-604 (2009). doi:10.1016/j.mcm.2008.03.013

9. Gasiński, L, Papageorgiou, NS: Nonsmooth Critical Point Theory and Nonlinear Boundary Value Problems. Chapman and hall/CRC Press, Boca Raton (2005)

10. Gasiński, L, Papageorgiou, NS: Nontrivial solutions for a class of resonant p-Laplacian Neumann problems. Nonlinear Anal. 71, 6365-6372 (2009). doi:10.1016/j.na.2009.06.039

11. Brezis, H, Nirenberg, L: Remarks on finding critical points. Commun Pure Appl Math. 44, 939-963 (1991). doi:10.1002/ cpa.3160440808

12. Rabinowitz, PH: Minimax methods in critical point theory with applications to differential equations. In CBMS Regional Conference Series in Mathematics, vol. 65,American Mathematical Soceity, Providence (1986)

doi:10.1186/1687-2770-2011-32

Cite this article as: Yang and Zhang: Existence results for a class of nonlocal problems involving p-Laplacian. Boundary Value Problems 2011 2011:32. 